\title{
P I I-02. In situ analysis by confocal microscopy of the cellular components of mucosal tissues within the framework of preclinical vaccine studies
}

\author{
CA Boggiano*1, AA Ginsberg1, MR Reynolds², NA Wilson², DI Watkins ${ }^{2}$ and \\ $\mathrm{AB}$ McDermott ${ }^{1}$
}

\author{
Address: ${ }^{1}$ Immunobiolgy - R\&D, International AIDS Vaccine Initiative, Brooklyn, NY, USA and ${ }^{2}$ Wisconsin National Primate Research Center- \\ Uniiversity of Wisconsin, Madison, WI, USA \\ * Corresponding author
}

from AIDS Vaccine 2009

Paris, France. 19-22 October 2009

Published: 22 October 2009

Retrovirology 2009, 6(Suppl 3):PI47 doi:10.II86/I742-4690-6-S3-PI47

This abstract is available from: http://www.retrovirology.com/content/6/S3/PI47

(c) 2009 Boggiano et al; licensee BioMed Central Ltd.

\section{Background}

The induction of a protective immune response against HIV remains elusive. In the majority of new HIV infections, virus traverses the mucosal barrier. A rapid and dramatic depletion of CD4+ T cells occurs in the gastrointestinal tract accompanied by active HIV replication. The Rhesus macaque/SIV model recapitulates mucosal transmission and pathogenesis, thus offering a means for a much-needed comprehensive surveillance of the innate and adaptive mucosal immune response during pre- and post-vaccination, and challenge. In preparation for preclinical studies we used mucosal tissues isolated from SIVinfected and uninfected Rhesus macaques to develop an integrated methodology to characterize by confocal immunofluorescence microscopy the gut associated lymphoid tissue (GALT), virus-specific immune responses and tissue architecture.

\section{Methods}

Necropsies or biopsies of duodenum, ileum and rectum were isolated from SIV-E660 infected or uninfected macaques. Tissues were fixed and cryopreserved embedded in OCT (optimum cutting temperature). Tissue sections $8 \mu \mathrm{m}$ thick were blocked and incubated with fluorochrome labeled antibodies targeting B and T-lymphocytes, natural killer cells, myeloid and plasmacytoid dendritic cells and immunoglobulins. SIV specific HLA- restricted mucosal $\mathrm{T}$ cell responses were monitored with fluorochrome labeled epitope specific tetramers.

\section{Results}

Data describing anatomical distribution of the different components of the adaptive and innate immune responses will be presented; emphasizing differences between infected and uninfected macaques.

\section{Conclusion}

These procedures will be included in upcoming preclinical protocols aiming to study tropism and immunogenicity of novel HIV vaccine candidates delivered by replicating viral vectors with mucosal tropism. Our methodology was developed using cross-reacting antibodies raised against human proteins yielding a system easily adaptable to human trials, in anticipation of clinical studies where mucosal immune responses are required for protection. 